\title{
Osificación heterotópica mesentérica precoz posabdomen abierto y su manejo quirúrgico. Presentación de un caso
}

\author{
Natalia Moreno B. ${ }^{1}$, Guillermo Reyes R. ${ }^{1}$, Ernesto Melkonian T. ${ }^{1}$ y Alejandro Readi V. ${ }^{1}$
}

'Equipo de Coloproctología Servicio de Cirugía, Hospital del Salvador. Santiago, Chile.

Recibido el 10 de enero de 2018 y aceptado para publicación el 19 de junio de

Correspondencia a: Dra. Natalia Moreno B. anatalia96@gmail.com

\section{Mesenteric heterotopic ossification after open abdomen and its surgical management. Presentation of a case}

Objective: Report the case of a patient with a history of multiple surgeries due to peritonitis and open abdomen, with intraoperative finding of mesenetrioc heterotopic ossification. Clinical case: A 59-year-old male patient with a history of complicated appendectomy 12 months ago, which requires the management of an open abdomen, right colectomy and terminal ileostomy. One year after appendectomy, is readmitted for transit reconstitution. Intraoperative finding were calcified mass in mesentery, of $15 \times 10 \times 6 \mathrm{~cm}$, whose histological study reports mesenteric heterotopic ossification. This entity has low frequency, and is associated with a history of trauma and abdominal surgery, is described as a cause of morbidity and mortality. Resective surgical management is feasible for experienced teams. Conclusion: A case with antecedent of open abdomen is described, with later finding of mesenteric heterotopic ossification. This clinical case is representative for its classic risk factors and management used for its resolution.

Key words: mesenteric heterotopic ossification; open abdomen; ossifying myositis.

\section{Resumen}

Objetivo: Reportar el caso de un paciente con antecedente de múltiples cirugías por peritonitis y abdomen abierto, con hallazgo intraoperatorio de osificación heterotópica en el mesenterio. Caso clínico: Paciente masculino de 59 años, con antecedente de apendicectomía complicada hace 12 meses, que en esa oportunidad requirió manejo de abdomen abierto, colectomía derecha e ileostomía terminal. Un año posapendicectomía reingresa para reconstitución de tránsito con hallazgo intraoperatorio de masa calcificada en mesenterio, de $15 \times 10 \times 6 \mathrm{~cm}$, cuyo estudio histológico informa osificación heterotópica mesentérica. Esta entidad es de baja frecuencia, asociada al antecedente de trauma y cirugía abdominal, y se ha descrito como causa de morbimortalidad. El manejo quirúrgico resectivo es factible por equipos con experiencia. Conclusión: Se describe un caso con antecedente de abdomen abierto, con posterior hallazgo de osificación heterotópica mesentérica. Este caso clínico es representativo por sus factores de riesgos clásicos y manejo empleado para su resolución.

Palabras clave: osificación heterotópica mesentérica; abdomen abierto; miositis osificante.

\section{Introducción}

La osificación heterotópica es una entidad poco frecuente y su etiología no ha sido completamente entendida. Generalmente, se asocia al antecedente de trauma abdominal o cirugía abdominal. Su aparición puede establecerse en semanas hasta años después de la cirugía y puede provocar síntomas con curso indolente o severo, y se ha descrito como causa de morbimortalidad, por lo que se ofrece cirugía en muchos casos, aunque no existe un consenso definitivo sobre la mejor alternativa terapéutica.

A continuación, presentamos un caso atendido en el Hospital del Salvador el año 2017. 


\section{Descripción del caso}

El caso clínico corresponde a un paciente de sexo masculino, de 59 años de edad, portador de ileostomía terminal, que ingresa al Hospital del Salvador para una cirugía de reconstitución de tránsito con técnica abierta.

El paciente tiene antecedentes de ser sano hasta hace 12 meses, momento en el cual fue operado de urgencia por un cuadro de apendicitis aguda complicada con perforación del apéndice y peritonitis localizada. En esa oportunidad se realizó una apendicectomía vía laparoscópica sufriendo una lesión térmica de asas de intestino delgado (íleon distal) detectada en el posoperatorio por clínica de peritonitis difusa y shock séptico de foco abdominal. Se realizó una reoperación de urgencia por técnica abierta donde se constatan lesiones térmicas cercanas a válvula ileocecal asociadas a necrosis de colon derecho, por lo que se decide realizar resección de $40 \mathrm{~cm}$ de íleon distal más hemicolectomía derecha. El paciente se manejó en Unidad de Paciente Crítico con abdomen abierto por 50 días durante los cuales requirió 5 aseos quirúrgicos. En el último aseo quirúrgico se decide realizar ileostomía terminal, abandono del cabo colónico y cierre de pared abdominal. El paciente evoluciona de forma satisfactoria y es dado de alta con abdomen cerrado e ileostomía terminal.

Una vez recuperado su estado general se estudia para reconstitución de tránsito con exámenes de sangre que no muestran alteraciones relevantes. No se realiza imagen abdominal preoperatoria. Por antecedentes descritos se planifica abordaje abierto. El paciente es sometido a una cirugía de reconstitución de tránsito por vía abierta, donde se realiza laparotomía media y disección roma de múltiples adherencias interasas. Luego de una laboriosa disección se descubre retracción de un segmento de asas de intestino delgado desde su mesenterio en cuya base, cercana a los vasos mesentéricos sin comprometerlos, se encuentra una masa pétrea, de forma irregular y espiculada, que mide $15 \times 10 \times 6 \mathrm{~cm}$ y que presenta adherencias firmes a asas de íleon distal por lo que se decide resección en bloque de la masa junto a $30 \mathrm{~cm}$ de intestino, respetando la irrigación mesentérica principal. Se reconstituye el tránsito mediante una ileotransverso anastomosis terminoterminal manual. Se cierra abdomen, se instalan drenajes tubulares y se cierra la herida de antigua ileostomía con jareta, completándose la cirugía sin incidentes. La pieza operatoria es enviada a estudio anatomopatológico diferido. El paciente evoluciona de forma satisfactoria en el período posoperatorio, es dado de alta a los 7 días. Inicialmente el paciente

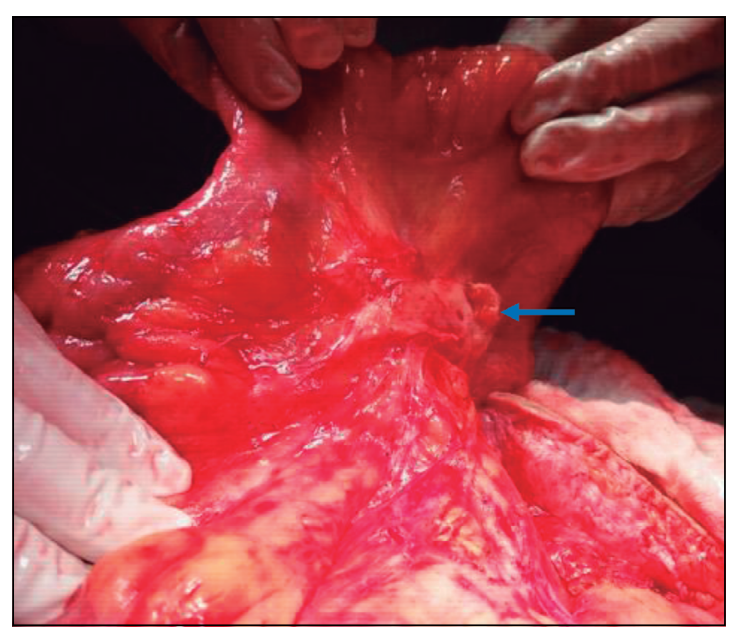

Figura 1. Imagen intraoperatoria, se observa retracción y engrosamiento del mesenterio secundario a la lesión pétrea en su raíz. Flecha azul: lesión en raíz mesentérica.

presenta diarrea, pero sus deposiciones cambian hasta volverse espesas con frecuencia defecatoria de 1-2 veces al día. El caso no presenta morbilidad alejada hasta la fecha. El informe de biopsia diferida de la pieza operatoria es compatible con osificación heterotópica mesentérica.

Se presentan fotografías intraoperatorias en las Figuras 1, 2 y 3 e imágenes histopatológicas en las Figura 4.

\section{Discusión}

Desde la primera mitad del siglo XX se han descrito diversos pseudotumores de partes blandas, benignos y malignos, de diferentes orígenes, siendo los de estirpe ósea de baja frecuencia. Los tumores osificados de partes blandas han recibido hasta el día de hoy diferentes nombres tales como miositis osificante o fascitis osificante ${ }^{1}$. La denominación de osificación heterotópica como tal fue descrita por primera vez por Riedel en 1883 y publicada por Askanazy en $1901^{2,3}$. El primer caso en la literatura sobre un tumor de estirpe ósea en el abdomen data de 1993 y corresponde a una lesión osificada próxima a la ileostomía de un paciente posproctocolectomía por colitis ulcerosa. Si bien los casos con osificación de cicatrices abdominales son relativamente frecuentes ${ }^{4,5}$, la localización intraabdominal de estas lesiones es poco frecuente y tiende a presentarse en el mesenterio ${ }^{6}$, por lo que en 1999 Wilson acuña el término propio de esta entidad como Osificación Heterotópica Mesentérica. Tres años antes de 


\section{CASOS CLÍNICOS}

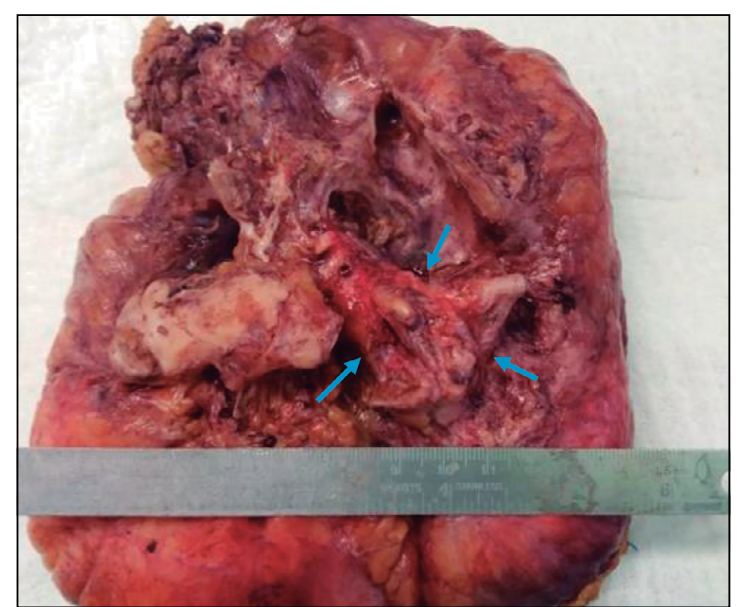

Figura 2. Pieza operatoria correspondiente a la resección en bloque del mesenterio y $30 \mathrm{~cm}$ de asas intestinales. Flechas: tumor en raíz mesentérica de $15 \times 10 \times 6 \mathrm{~cm}$.

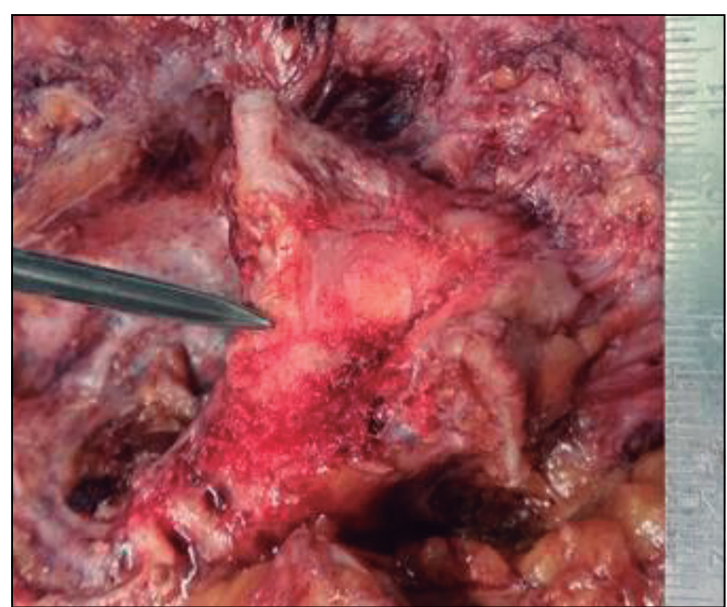

Figura 3. Tumor pétreo seccionado, se observa su forma irregular y espiculada.

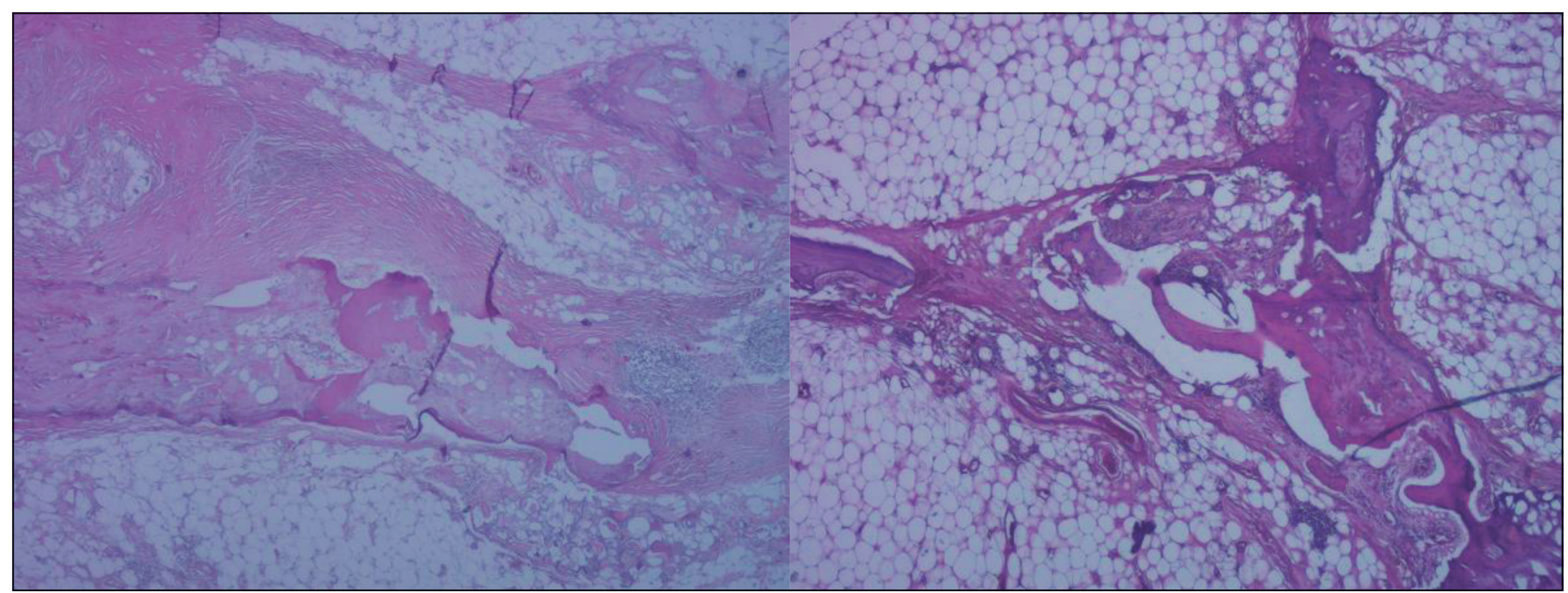

Figura 4. Fotomicrografía de osificación heterotópica de tejido mesentérico. Se observan trabéculas de hueso por osteoblastos que forman osteoide (material eosinofílico) y osteomas primitivos.

acuñarse el término, se realiza el primer reporte en Chile, cuando se presenta un caso denominado osificación difusa intraabdominal como complicación de colitis ulcerosa ${ }^{7}$.

La osificación heterotópica corresponde a lesiones por depósito de calcio y osteoblastos que forman capas óseas, en ausencia de características de malignidad en las células, lo que las diferencia de los osteosarcomas. Estos focos de osificación pueden presentarse prácticamente en cualquier zona de tejidos blandos, incluso dentro de otras neoplasias como el cáncer de colon o pólipos ${ }^{8}$. Su etiología exacta aún es desconocida ${ }^{9}$, pero hay varias teorías propuestas que explican la fisiopatología de la osificación del tejido mesentérico. Una de las teorías propone la presencia de células precursoras osteogénicas en los tejidos mesentéricos, que al ser estimuladas en un ambiente nutricional propicio pueden producir osificación del tejido ${ }^{10}$. Por otro lado, se piensa que hay sustancias secretadas por las células epiteliales capaces de inducir osteogénesis durante el proceso de reparación de los tejidos. Este proceso puede ocurrir de forma difusa intraabdominal o focalizada (lo más frecuente) y dentro o fuera de las asas intes- 
tinales. En los casos de OHM en que hay compromiso transmural del tubo digestivo el recto parece ser el segmento más comprometido. Algunos casos comprometen la herida quirúrgica, donde se postula un origen óseo de las células precursoras migrantes desde un hueso dañado, como la apófisis xifoides y el pubis hasta la herida ${ }^{4}$.

En la mayoría de los casos de OHM descritos en la literatura se trata de pacientes varones que han sufrido trauma abdominal o que han sido sometidos a múltiples cirugías abdominales, en un contexto de cuidados intensivos ${ }^{1,6,8,11}$. Algunos estudios han intentado determinar si otros factores, diferentes a la cirugía, se asocian a la aparición de OHM en estos pacientes y se ha reportado una asociación con la nutrición parenteral, postulando que favorece el proceso, aunque no es un factor determinante ni necesario para la osificación.

La presencia de una masa de osificación heterotópica mesentérica puede provocar síntomas gastrointestinales y abdominales donde el más frecuente es el dolor abdominal, sin embargo, hay casos reportados de obstrucción intestinal y mortalidad ${ }^{12}$. La aparición de los síntomas es variable, pudiendo iniciar en las primeras semanas del posoperatorio, hasta años después. El proceso puede recurrir con laparotomías repetidas. En el estudio de estos pacientes no hay exámenes séricos específicos, aunque se puede encontrar niveles elevados de fosfatasa alcalina, el marcador CA-125 y la paratohormona. Las imágenes suelen ser de mayor utilidad; la radiografía mostrará elementos radiopacos, heterotópicos irregulares y la tomografía computarizada puede mostrar un patrón de corteza y trabéculas óseas bien definidas que son sugerentes del diagnóstico, sin embargo, se debe interpretar de forma cuidadosa y no confundir con cuerpos extraños o con extravasación del medio de contraste ${ }^{6}$. El estudio histopatológico de la lesión busca la presencia de zonas reactivas, capa osteoídea gruesa, hueso trabecular maduro y la ausencia de atipia nuclear, necrosis y figuras mitóticas atípicas, esto último es fundamental para descartar el osteosarcoma extraesquelético, en cambio la alta celularidad y las mitosis frecuentes no permiten diferenciar entre ambas entidades diagnósticas, ya que la OHM puede presentarlas.

Debido a lo infrecuente de esta patología el tratamiento óptimo no está claro. La tendencia actual es el tratamiento quirúrgico, a pesar del riesgo de recurrencia. La elección de resecar las masas está fundamentado en los síntomas persistentes, el riesgo de complicaciones graves y la falta de evidencia de la seguridad del manejo conservador a largo plazo, aunque actualmente, no hay un consenso ni estudios aleatorizados que hayan demostrado cual es el tratamiento de elección, principalmente por el número reducido de $\operatorname{casos}^{9}$. Cabe señalar que la resección quirúrgica no es fácil y debe ser realizada por cirujanos con experiencia. Algunas de las dificultades técnicas se deben a la forma irregular de la masa osificada, generalmente muy adherida al tejido circundante, el gran tamaño alcanzado por algunas piezas o el gran número de pequeños focos osificados que deben resecarse por separado ${ }^{13,14}$.

El manejo conservador inicial puede ser justificado en casos que no requieran cirugía de urgencia. Como tratamiento complementario se ha propuesto la indometacina como antiinflamatorio de elección por su efecto de inhibición de la osificación ${ }^{13}$ aunque su rol en OHM y su efectividad como profilaxis no están totalmente definidas. En casos de hiperparatiroidismo asociado se ha usado cimetidina que contribuye a normalizar los niveles de calcio y $\mathrm{PTH}^{15}$. Los bifosfonatos y la radioterapia también han sido utilizados.

El caso clínico presentado se trata de un paciente que cumple con los factores de riesgo clásicos asociados a la OHM, tales como el manejo de abdomen abierto, múltiples cirugías abdominales y el uso de nutrición parenteral en el contexto del cuidado intensivo. Este caso presentó algunos síntomas gastrointestinales, como aumento de frecuencia defecatoria y menor consistencia de las heces las primeras semanas posoperatorias, pero esto se atribuyó a la resección intestinal realizada y la adaptación lenta de su colon desfuncionalizado previamente. El dolor abdominal descrito en la literatura tiende a ser inespecífico, pero persistente o recidivante, situación que no presentó el paciente, sin embargo, sólo pudo observarse 1 año, ya que luego fue sometido a una nueva laparotomía con resección de la masa. Tampoco contamos con imágenes preoperatorias que muestren la masa osificada ni contamos con evidencia del tiempo en que se comenzó a osificar el tejido. Cabe destacar que la re-laparotomía es un factor de riesgo para recidiva de las lesiones de $\mathrm{OHM}$ por lo que se sugiere al paciente someterse a controles clínicos y/o imagenológicos a mediano y largo plazo, por este riesgo.

\section{Conclusión}

La osificación heterotópica mesentérica es una entidad patológica de baja frecuencia. La mayoría de los casos se asocian a trauma quirúrgico previo y conforman masas localizadas que pueden provocar síntomas. El manejo quirúrgico es complejo, pero 
factible. El caso clínico presentado conforma un ejemplo clásico de los factores de riesgos descritos en la literatura.

\section{Responsabilidades éticas}

Protección de personas y animales. Los auto- res declaran que para esta investigación no se han realizado experimentos en seres humanos ni en animales.

Confidencialidad de los datos. Los autores declaran que en este artículo no aparecen datos de pacientes.

Conflictos de interés: no hay.

\section{Referencias}

1. Zamolyi R, Souza P, Nascimento A, Unni K. Intraabdominal Myositis Ossificans: A Report of 9 New Cases. Int J Surg Pathol. 2006;14:37-41.

2. González Q, Bahena-Aponte J, Lezama de Luna J, Ramírez-Avilés E. Metaplasia ósea distrófica en el mesenterio de intestino delgado en un paciente con múltiples cirugías abdominales. Informe de un caso. Rev Gastroenterol Mex. 2011;76:251-4.

3. Shehab D, Elgazzar A, Collier B. Heterotopic ossification. J Nucl Med. 2002;43:346-53.

4. Berna J, González-Valverde M, GarcíaMedina V, Aleman P, Madrigal M. Heterotopic Ossification of Abdominal Wall Scar. Chir Gastroenterol. 2001;17:340-1.

5. Reardon M, Tillou A, Mody D, Reardon P. Heterotopic calcification in abdominal wounds. Am J Surg. 1997;173:145-7.
6. Binesh F, Akhavan A, Navabii H, Ostadi M. Heterotopic mesenteric ossification: report of a case and review of the literature. BMJ Case Reports 2012; doi:10.1136/bcr-02-2012-5793.

7. Jensen C, Marine L, Vergara J, Azolas C, Andrade L, Pérez G, et al. Osificación mesentérica. Complicación en colitis ulcerosa idiomática. Rev Chil Cir. 1996;48:516-18.

8. Alper M, Akyürek N, Patiroglu T, Yüksel O, Belenli O. Heterotopic bone formation in two cases of colon carcinoma. Scandinavian Journal of Gastroenterology 2000;35:556-8.

9. Schiergens T, Reichelt A, Thasler W, Rentsch M. Abdominal bone formation. J Gastrointest Surg. 2015:19;579-80.

10. Kaplan F, Glaser D, Hebela N, Shore E. Heterotopic ossification. J Am Acad Orthop Surg. 2004;12:116-25.

11. Honjo $H$, Kumagai $Y$, Ishiguro $T$, Imaizumi $\mathrm{H}$, Ono $\mathrm{T}$, Suzuki $\mathrm{O}$, et al. Heterotopic mesenteric ossification after a ruptured abdominal aortic aneurism: Case report with a review of literatures. Int Surg. 2014;99: 479-84.

12. Haque S, Eisen R, West A. Heterotopic bone formation in the gastrointestinal tract. Arch Pathol Lab Med. 1996;120:666-70.

13. Torgersen Z, Osmolak A, Bikhchandani $\mathrm{J}$, Forse A. Ectopic bone in the abdominal cavity: A surgical nightmare. J Gastrointest Surg. 2013;17:1708-11.

14. Del Pozzo J, Soto B, Hernández D, Mata C, Luna J. Manejo quirúrgico de obstrucción intestinal secundaria a osificación heterotópica mesentérica. Informe de un caso. Cir Gen. 2006: 28;248-52.

15. Yamamura-Idei $Y$, Kitazawa $S$, Kitazawa R, Fujimori T, Chiba T, Maeda S. Parathyroid hormonerelated protein in gastric cancers with heterotopic ossification. Cancer 1993:72;1849-52. 